\title{
Diabetes y pie diabético: una problemática mundial abordada desde la fisioterapia
}

\author{
Diabetes and diabetic foot: a world problem addressed from physiotherapy
}

Arroyo $L D^{1}$, Burbano $J I^{2}$.

${ }^{1}$ Fisioterapeuta, Especialista en Docencia Universitaria.

Magíster en Educación y Desarrollo Humano.

Docente, Universidad Santiago de Cali, Cali, Colombia.

${ }^{2}$ Estudiante de Fisioterapia, Universidad Santiago de Cali, seccional Palmira.

Líder, Semillero de Investigación Constructores de Conocimiento. Joven investigador, USC.

Representante, Asociación Colombiana de Estudiantes de Fisioterapia (ACEFIT), Regional Pacífico.

Autor de correspondencia: Luz Dary Arroyo Valencia

Correo electrónico: luz.arroyo00@usc.edu.co

Fecha de recepción: 17/06/2018

Fecha de aceptación: 11/04/2019

\section{Resumen}

El presente escrito indaga sobre el papel del fisioterapeuta en la atención del paciente con diabetes y pie diabético. Para ello, se hizo una búsqueda de la literatura en bases de datos virtuales conocidas, usando palabras clave y análisis de la información. Se encontró poca participación de los fisioterapeutas en el área preventiva y escasez de programas educativos sobre el problema de la diabetes y del pie diabético. No obstante, los procesos de rehabilitación son fuertes, lo que logra la inclusión de la persona en diferentes ámbitos, usando como estrategia principal el ejercicio físico. Continúan faltando las actividades de prevención del pie diabético.

Palabras clave: diabetes mellitus, ejercicio, pie diabético, fisioterapia.

\section{Abstract}

The present document investigates the physiotherapist role in the care of the patient with diabetes and diabetic foot. For this purpose, literature was searched in known virtual databases, using key words and information analysis. There was little participation of physiotherapists in the preventive area and lack of educational programs on the problem of diabetes and diabetic foot. However, the rehabilitation processes are strong, achieving the inclusion of the person in different areas, using as main strategy the physical exercise, the activities of prevention of diabetic foot are still lacking.

Key words: diabetes mellitus, exercise, diabetic foot, physical therapy.

\section{Introducción}

La diabetes se considera una problemática de salud pública a nivel mundial, que afecta a hombres y mujeres sin distinción de raza o condición social, generadora de discapacidad que altera la calidad de vida de las personas y su familia. Actualmente, hay más de 400 millones de personas diagnosticadas con diabetes y se prevé que para el año 2030 serán aproximadamente más de 500 millones, lo que la convierte en una entidad de intervención prioritaria no solo en el sistema de salud, sino para otros sectores de la sociedad ${ }^{(1)}$.

Dentro de las complicaciones más frecuentes de la diabetes se encuentran la retinopatía diabética, ceguera, incremento del riesgo cardiovascular y, por ende, aumento de la probabilidad de accidente cerebrovascular y falla renal ${ }^{(2)}$. Además, se producen alteraciones del sistema nervioso que comprometen la salud en general y afectan la calidad de vida del individuo.

$\mathrm{Al}$ pie diabético se le considera la principal complicación dada principalmente por las neuropatías y vasculopatías presentes en el paciente diabético y generadora de ulceración en el mismo. Su prevalencia está entre el 8\% y el 13\% de los pacientes diabéticos y afecta mayormente a adultos entre los 45 y 65 años, los cuales se encuentran en etapa laboral y socialmente activa ${ }^{(3)}$.

El pie diabético es responsable de amputación en miembros inferiores y generadora de limitación funcional en el individuo con diabetes, lo que compromete su desempeño y participación social. Su prevalencia y costo de atención causa un gran impacto en los sistemas de salud, lo que la convierte en una problemática que debe ser abordada desde la prevención primaria, la cual debe ser dirigida por un equipo multidisciplinario.

El manejo de este problema se enfoca en mantener el control de la glucemia y evitar la aparición de complicaciones. 
Las estrategias incluyen prevención, educación de pacientes y prestadores de servicios de salud, tratamiento de complicaciones y monitorización estrecha de la problemática. No obstante, son escasos los estudios que hablan de la participación del fisioterapeuta (encargados del movimiento corporal humano) en el manejo de esta situación. Estos profesionales a través del ejercicio físico y educación para la salud pueden contribuir en la reducción de los estados de morbilidad y mortalidad en pacientes diabéticos y con pie diabético.

Se hablará entonces, en primer lugar, de la diabetes y su impacto en la salud física de la persona; en segundo lugar, del pie diabético como problema responsable de amputación y alteración del esquema corporal y de la funcionalidad del individuo, además de los procesos de rehabilitación del mismo; finalmente, se intentará plasmar el papel del fisioterapeuta en el manejo del pie diabético.

\section{Generalidades de la diabetes}

La diabetes es una de las enfermedades de carácter metabólico, no trasmisible, caracterizada por el aumento de los niveles de glucosa en sangre (hiperglucemia) a causa del disfuncionamiento del páncreas, responsable de la secreción de la insulina transportadora del azúcar a las células ${ }^{(4)}$. Es un síndrome crónico incurable e irreversible, pero tratable, que conduce a un proceso degenerativo del ser humano y que conlleva a complicaciones que pueden producir la muerte o condiciones que afectan la calidad de vida no solo de quien vive con diabetes, sino también de su entorno familiar y social.

Ahora bien, la diabetes es considerada una problemática de salud en todo el mundo. Según datos de la Organización Mundial de la Salud (OMS), su prevalencia ha aumentado del $4,7 \%$ en 1980 al 8,5\% en el año 2014 y será la séptima causa de mortalidad en $2030^{(5)}$. Esta enfermedad no transmisible y de carácter metabólico es responsable de un alto número de muertes en el mundo. Para el año 2012 fue la octava causa de muerte en el mundo y es culpable de 1,5 millones de muertes cada año. Otros 2,2, millones de muertes están vinculadas a los altos niveles de azúcar en la sangre, en donde el $43 \%$ de estos (3,7 millones de personas) murió antes de cumplir los 70 años ${ }^{(6)}$ En Colombia, según el Observatorio Nacional de Salud (ONS), la diabetes alcanzó una tasa de mortalidad promedio entre 2009 y 2011 de 10,4 por 100000 habitantes, ubicándose dentro de las primeras 10 causas de mortalidad general, siendo más del $50 \%$ prevenibles ${ }^{(7)}$.

Por otro lado, los pacientes diabéticos consumen el $11 \%$ del presupuesto total de salud en los Estados Unidos y alrededor del 6\% del costo en salud en Latinoamérica y el Caribe, lo que la hace una entidad de interés en salud pública, además del impacto que genera la alta carga en los indicadores de morbilidad y mortalidad en salud ${ }^{(8)}$. En Colombia, el trabajo realizado por Tamayo, y presentado por el Observatorio de Diabetes en
Colombia (ODC), muestra los datos para el 2007 del costo anual de la atención de la diabetes mellitus (DM) controlada por paciente, sin contemplar el escenario de las complicaciones, esta correspondió a \$2 438 000, que ascienden a \$7 166000 cuando la farmacoterapia incluye insulina, lo que la convierte en una realidad preocupante y de alto impacto socioeconómico ${ }^{(9)}$.

En Colombia, en 2013 el 75,58 \% (479 238) de los casos de diabetes mellitus se encuentran afiliados al régimen contributivo, el 20,75 \% (131 554) al subsidiado y el restante 3,68 \% (23 305) a las fuerzas militares. Alrededor del $70 \%$ de las personas mayores de 30 años diagnosticadas pertenece al régimen contributivo, a la vez que la mayor proporción de personas menores de 20 años está afiliada al subsidiado. Los porcentajes más altos de casos de diabetes se presentaron en el Distrito Capital, con un 20,20 \% (128 067); en Antioquia, con un 18,11 \% (114 848) y en el Valle del Cauca, con un 14,57 \% (92 406) ${ }^{(10)}$.

Por esta razón, en Colombia se han realizado estudios para conocer los costos de la diabetes, como el estudio realizado por el Dr. Martín Romero, economista de la salud, y publicado en Value in Health (2017), titulado Impacto económico de la hipoglucemia en Colombia, en el que se estima que el valor de una hipoglucemia grave le cuesta al sistema 2,2 veces el valor de la unidad de pago por capitación (UPC), lo cual es el valor asignado por el gobierno a cada uno de los afiliados al sistema de salud para cubrir todas las prestaciones de servicios en salud; por otro lado, la hipoglucemia moderada está costando solo el 0,5 del valor de una UPC. Por su parte, en el costo de la hipoglucemia grave se identifica que los costos por hospitalización son aproximadamente el valor de una UPC, mientras que los costos relacionados con la atención por el servicio de urgencia son de aproximadamente el $20 \%$ de una UPC. Partiendo del costo calculado para los dos tipos de hipoglucemia, se calcula un costo anual de USD 562474 para cerca de 1000 pacientes ${ }^{(11)}$.

De igual manera, es importante recordar que la diabetes, por ser una enfermedad multiorgánica, afecta el funcionamiento de diversos sistemas, como el neurológico y el vascular, principalmente, involucrados en la generación del movimiento corporal humano, como un elemento esencial para el desarrollo de las actividades de la vida diaria, pilar fundamental de la supervivencia y el desempeño del hombre en la sociedad donde se desenvuelve. En un estudio descriptivo observacional realizado en la Plata, Argentina, cuyo objetivo fue estimar y caracterizar el impacto de la enfermedad sobre la productividad laboral de las personas con diabetes entre los 18 y 75 años de edad, usando como instrumento el cuestionario WPAI-GH (work productivity and activity impairment - General Health version) mostró que la diabetes también disminuyó un $25 \%$ la capacidad para realizar actividades regulares diarias y que afecta más a mujeres que a hombres (30\% frente a $20,3 \%$, respectivamente), esto no solo altera el componente biológico, sino el componente psicosocial del individuo ${ }^{(12)}$. 
Entonces, para el tratamiento de la diabetes, por ser un problema multifactorial, debe ser tratado por un equipo multidisciplinar, que se encargue, desde una mirada integral, en controlar los niveles de azúcar en sangre, modificar los factores de riesgo y prevenir las complicaciones de la diabetes. Para ello se hace necesario recordar que existen 2 modalidades de tratamiento: el farmacológico y el no farmacológico. Dentro del primer grupo se encuentran todos los medicamentos hipoglucemiantes o antidiabéticos orales y la insulina, los cuales se encargan de normalizar el azúcar y mejorar la cantidad de insulina; el segundo grupo consideramos que es el más importante, pues está demostrado que las estrategias, como la prescripción adecuada del ejercicio aeróbico y de fuerza en el diabético, es un pilar fundamental en su manejo, tratamiento, control y pronóstico y tiene múltiples beneficios no solo en el control glucémico, sino también en parámetros cardiovasculares, metabólicos, antropométricos, psicosociales e incluso en la mortalidad ${ }^{(13)}$. De igual forma, el tratamiento no farmacológico involucra la educación sobre autocuidado basado en el autoanálisis de niveles de glucosa y orina en sangre, cuidado de los pies, higiene, tratamiento dietético e intervención temprana para reducir las complicaciones.

Una de las complicaciones más frecuentes de la diabetes son las úlceras y amputación de los miembros inferiores, generado principalmente por la neuropatía y las vasculopatías. Aunque existe una clasificación propuesta por Silva y colaboradores, la cual tiene en cuenta el compromiso de la circulación, estas son complicaciones macrovasculares, como la enfermedad arterial coronaria, enfermedad cerebrovascular y vascular periférica, y las microvasculares, como la retinopatía, nefropatía y neuropatía, las cuales aumentan la morbilidad y mortalidad asociada con otros factores, como la hipertensión arterial, tabaquismo y dislipidemia ${ }^{(5)}$.

Finalmente, la diabetes es considerada la enfermedad del siglo XXI y el pie diabético una de sus complicaciones más frecuentes. Según la American Diabetes Association, alrededor del $15 \%$ de las personas con diabetes desarrollará una lesión en sus pies a lo largo de su vida y cada año cuatro millones de personas desarrollarán una úlcera en el pie ${ }^{(8)}$.

\section{Pie diabético (PD)}

Cuando se habla de PD se refiere a una de las complicaciones tardías y quizás devastadora que presenta la persona diabética. Esta es una lesión degenerativa de los tejidos y su función debido a la enfermedad vascular o a la disfunción del nervio periférico; caracterizada por la aparición de ulceración, infección o destrucción de tejidos profundos del pie. Su prevalencia está entre el $8 \%$ y $13 \%$ de los pacientes diabéticos y afecta mayormente a los adultos entre los 45 y 65 años de edad, los cuales se encuentran aún en etapa laboral y socialmente productiva, esto afecta su calidad de vida y la de la familia ${ }^{(3)}$.
Según Bakker y colaboradores, la probabilidad de que una persona diabética pierda una extremidad inferior es 40 veces más que las personas que no padecen la enfermedad ${ }^{(14)}$. Esto acarrea consecuencias humanas y económicas bastante altas, pues la persona amputada altera su esquema corporal, afecta su patrón de marcha y la realización de actividades de la vida diaria que, si bien, con un proceso de rehabilitación algunas personas podrán incluirse nuevamente en la sociedad, otros quedan con una discapacidad. Además, se encuentran los costos económicos elevados que el pie diabético genera al sector salud, pues un paciente con esta enfermedad puede requerir una hospitalización prolongada, tratamientos y exámenes diagnósticos costosos (antibióticos, cirugía vascular, curaciones diarias, Doppler, servicio de rehabilitación, entre otros), lo que obliga a los profesionales de la salud a buscar medidas costo efectivas para reducir su prevalencia e incidencia en la población afectada de diabetes.

La diabetes es la principal causa de amputación no traumática de miembros inferiores, precedida generalmente por úlceras en el pie. La identificación de los pacientes en riesgo es el primer paso para prevenir la amputación, seguida de la educación al paciente y su familia. Sin embargo, en nuestro contexto son escasos los estudios que desde fisioterapia hablan de las actividades de prevención del pie diabético y con un enfoque de atención primaria en salud.

Ahora bien, preguntémonos ¿Por qué pasa el pie diabético? La literatura reporta que existen unos factores predisponentes, desencadenantes y agravantes, los cuales se describen a continuación, pues el conocimiento de ellos es una base para la prevención del pie diabético.

- Factores predisponentes: son aquellos que en un enfermo diabético van a ocasionar riesgo de sufrir lesión. Estos están relacionados, por un lado, con la isquemia generada por la vasculopatía responsable de los cambios iniciales, como la sequedad de la piel, lo que favorece la aparición de úlceras ante cualquier traumatismo; y, por otro lado, con la neuropatía, pues el principal síntoma objetivable en los pacientes es la disminución de la sensibilidad profunda, táctil, superficial y térmica, lo que altera el sistema musculoesquelético (aparecen dedos en garra) y provoca restricción de la movilidad articular

- Factores desencadenantes o precipitantes: factores que hacen debutar la lesión. Estos pueden ser extrínsecos o intrínsecos. Los extrínsecos se dividen según la causa en traumáticos (golpes, caídas); mecánicos, como el calzado mal ajustado, considerado uno de los principales aspectos a tener en cuenta en la evaluación del pie diabético, pues es el responsable de más del $40 \%$ de la aparición de úlceras; térmico, al introducir los pies en agua caliente, uso de mantas eléctricas o bolsas calientes; químicos, uso de agentes queratolíticos, especialmente los que contienen ácido salicílico; e intrínsecos, que incluyen las deformidades (hallux valgus o juanete, dedos en garra, artropatía de 
Charcot, entre otros) que pueden generar un aumento de la presión plantar, lo que ocasiona callosidades, las cuales pueden terminar en lesiones preulcerosas.

- Factores agravantes o perpetuantes: factores que en un pie diabético establecido van a facilitar la aparición de complicaciones y retrasar la cicatrización. Aquí encontramos los microorganismos de diversa índole, en su mayoría de naturaleza saprófita, como estafilococos, estreptococos, entre otros. También pueden hallarse si las úlceras son lo suficientemente profundas, organismos aerobios y anaerobios como Escherichia coli y Clostridium perfringens. Tales microorganismos pueden llegar a invadir los tejidos profundos y ocasionar cuadros como celulitis y artritis séptica ${ }^{(15)}$.

Las personas que presenten uno o más de estos factores deben ser controladas cada 6 meses por un equipo multidisciplinar, quienes con medidas, como la educación al paciente y la familia, disminuyen los riesgos de presentar pie diabético y, por ende, las probabilidades de amputación de miembros inferiores, que no solo es "retirar la extremidad comprometida", sino las implicaciones que la misma trae a nivel psicológico por alteración de su esquema corporal y patrón de marcha, que si bien se puede solucionar en algunos casos con las prótesis, las alteraciones neurológicas que presenta el paciente a causa de la diabetes dejan limitaciones en la realización de algunas de las actividades de la vida diaria que impliquen desplazamiento y cargas de peso mayores, lo que afecta en últimas el bienestar y la calidad de vida.

En un estudio descriptivo transversal realizado en Bogotá en el año 2009-2011, las actividades más usadas por el personal de salud (médicos y especialistas) y los pacientes para la prevención de la diabetes mellitus y el pie diabético son aquellas relacionadas con el cambio en el estilo de vida asociados con la nutrición. El asistir al control de forma periódica (cada mes el 53,8\% y entre 2 y 3 meses el 40,0\%) fue una de las acciones más frecuentes de los pacientes para prevenir la aparición de complicaciones por DM, aunque en las consultas los médicos omitieron preguntas importantes sobre la presencia de síntomas, como disestesias y claudicación intermitente asociados con la vasculopatía y neuropatía responsables del pie diabético en la gran mayoría de los casos. Sin embargo, el $40,5 \%$ de los pacientes participantes del estudio refirió recibir recomendaciones por parte del médico tratante sobre el autocuidado de los pies.

Por otra parte, los fisioterapeutas atendemos frecuentemente en los servicios de rehabilitación personas amputadas por diabetes, quienes en su mayoría no recibieron información sobre las medidas preventivas para evitar su aparición y terminaron con lesiones ulcerativas, las cuales son la principal causa de degeneración de los tejidos del pie, tal como lo demuestra el estudio descriptivo transversal mencionado anteriormente sobre las actividades de prevención y factores de riesgo de la DM y PD, donde médicos y especialistas no cuen- tan con una evaluación integral del paciente, por tanto, son mínimas las actividades de prevención de aparición de PD ${ }^{(16)}$.

La prevención del pie diabético requiere del control de todos los factores de riesgo: peso corporal, glucemia, hipertensión arterial y dislipidemia, los cuales desde fisioterapia con un programa de ejercicios aeróbicos, fuerza y flexibilidad principalmente de forma regular y de intensidad moderada, ayudan a mejorar el bienestar y pronóstico del paciente, además de optimizar la condición física y funcionamiento del organismo, lo que incrementa la calidad de vida y reduce la mortalidad en personas con DM o sin ella, lo que lo hace un elemento esencial de la promoción de la salud ${ }^{(17)}$.

De igual manera, los fisioterapeutas cuando atienden personas amputadas por diabetes se basan en un tratamiento global y dinámico que comienza desde el momento de la lesión y continua hasta la incorporación del paciente en las actividades de la vida diaria, en compañía de otros profesionales. Cuando no se identifica a tiempo en la consulta los riesgos que tiene la persona diabética de desarrollar un pie diabético y la misma no cuenta con la información necesaria y suficiente sobre los cuidados generales de la diabetes y la forma de prevenir sus complicaciones agudas o crónicas, esta aumenta los riesgos de padecerlas.

\section{¿Cómo se trata el pie diabético?}

Siendo la DM y el PD una enfermedad epidémica con grandes repercusiones en el sistema de salud y la sociedad en general, en Colombia, el Ministerio de Salud y Protección Social presentó en su Guía de atención de la diabetes mellitus tipo 2 un enfoque de tratamiento integral, el cual involucra la educación como un elemento esencial en el manejo de la misma. Es así como dice que "el tratamiento inicial de todo paciente diabético está dirigido a la obtención de un óptimo nivel educativo que le permita tener los conocimientos necesarios para poder llevar a cabo un buen control metabólico de la enfermedad dirigido a la prevención de las complicaciones agudas y crónicas dentro de los mejores parámetros de calidad de vida posibles" (18). Esta propuesta incluye la adquisición de un hábito alimenticio adecuado y el incremento de la actividad física como estrategias fundamentales en el control de la glucemia y la prevención de alteraciones cardiovasculares.

La guía también incluye el tratamiento de la principal complicación de la diabetes, el pie diabético, abordada desde las 3 estrategias de prevención de la siguiente manera: en la prevención primaria se busca la detección temprana del pie en riesgo usando la educación sobre calzado, higiene podológica y prevención del trauma como medio esencial en la valoración; la revisión diaria de los pies ha sido una actividad que permite la toma de conciencia y generación de la cultura de autocuidado. Tal como lo reporta Pinilla y colaboradores en su investigación sobre los factores de riesgo en diabetes mellitus y pie diabético: un enfoque hacia la prevención primaria, donde en 
una revisión sistemática de Cochrane se evaluó el desenlace clínico con estrategias educativas variadas y con tiempo de duración distinto. En este estudio se reportó que el paciente que recibe educación mejora su conocimiento sobre los aspectos básicos del pie diabético y de autocuidado, lo que conlleva a reeducación de complicaciones como úlcera o amputación ${ }^{(19)}$.

Ahora bien, como fisioterapeutas, la educación del paciente es un pilar fundamental en los procesos de intervención en salud pública con el fin de prevenir aquellas conductas que representan riesgo para la salud o fomentar las que requiere la persona en el proceso de tratamiento de su enfermedad o rehabilitación, lo que implica una mirada integral de las personas en los diferentes entornos donde viven, así como la participación en un equipo de trabajo interdisciplinario e intersectorial que aporte a las transformaciones socioculturales, económicas y políticas del individuo y la comunidad. Sin embargo, son escasos los estudios en nuestro contexto que reportan experiencias desde la fisioterapia en la educación para salud y más en el caso de la diabetes y pie diabético ${ }^{(20)}$.

En cuanto a la prevención secundaria, la intención es evitar el desarrollo de gangrena mediante el cuidado adecuado de las úlceras y corrección de los factores desencadenantes (aliviar los puntos de presión, calzado ortopédico, manejo podológico de las deformidades ungueales, riego sanguíneo, entre otros). En la prevención secundaria se busca detectar, cuidar y tratar precozmente las alteraciones manifestadas en el pie; ellas dependerán de la cronicidad de la enfermedad y de los cuidados realizados por los pacientes recibidos en procesos educativos específicos. Es importante destacar que la educación debe ser impartida por un grupo de profesionales que conozcan y manejen la diabetes y las complicaciones como el pie diabético, por lo que surge la necesidad de que el pie diabético sea valorado y tratado de forma multidisciplinaria, la cual no es frecuente en muchos contextos, cuando una forma estructurada se justifica tanto por la optimización de los recursos en su fase diagnóstica como por la racionalidad, operatividad, eficacia y eficiencia en su fase terapéutica, a menudo compleja y de morbilidad elevada ${ }^{(21)}$.

De igual manera, los trabajos realizados en torno al manejo del pie diabético básicamente se enfocan en el tratamiento de la ulceración, pues este es el principal factor de riesgo de amputaciones no traumáticas en diabéticos, con uso de estrategias que controlen y traten los múltiples factores causantes de la ulceración severa del pie diabético concomitante con el desbridamiento, control de la infección y revascularización, que son los pasos clave para la estabilización de la herida y su preparación para una reconstrucción exitosa ${ }^{(22)}$.

Por último, está la prevención terciaria con el fin de evitar la amputación. Para esta debe intervenir un equipo multidisciplinario especializado y suele requerir tratamiento intensivo intrahospitalario. La amputación es la opción terapéutica facilitada por la extensa necrosis tisular en el pie o cuando diversas alternativas terapéuticas han fracasado. De esta forma, la diabetes es la principal causa de amputaciones de extremidades inferiores no traumáticas, siendo la responsable de aproximadamente el $50 \%$ de las mismas. Es posible que este problema se acentúe en el futuro, ya que la incidencia de la DM se encuentra en franco aumento en los países en vías de desarrollo, probablemente debido a cambios en los hábitos alimentarios y al sedentarismo. Esto, más la prolongación de la expectativa de vida, con el consiguiente envejecimiento de la población, incrementará inevitablemente la prevalencia de DM y de sus complicaciones crónicas ${ }^{(23)}$.

La guía no presenta el papel de cada profesional de la salud en el manejo de la DM tipo 2; sin embargo, propone el ejercicio físico, definido este como toda actividad física regular que se realice mínimo 3 veces por semana y que produzca un mayor consumo de calorías. El ejercicio debe ser aeróbico (caminar, nadar, trotar, entre otros) de intensidad moderada y de duración igual o mayor de 30 minutos ${ }^{(24)}$. Es aquí donde el fisioterapeuta cobra importancia, pues como profesionales utilizamos la prescripción del ejercicio físico, estrategia principal para la promoción de la salud y bienestar cinético, prevención de alteraciones del movimiento y manejo de la disfunción del mismo ocasionada por la diversidad de enfermedades, que para el caso de la diabetes mellitus y el pie diabético existen diversos artículos que evidencian sus efectos positivos, tal como lo demuestra la revisión sistemática de la literatura que habla de los efectos del ejercicio en el control glucémico, usando diversas modalidades de ejercicio, como el aeróbico, ejercicios de fuerza, programa de entrenamiento interválico y de combinación de modalidades de entrenamiento a largo plazo, que muestran eficacia en el control glucémico tanto por el entrenamiento a largo plazo como en las 24-48 horas postentrenamiento. El seguimiento de un plan de entrenamiento estructurado es la clave para la mejora ${ }^{(25)}$.

Por otra parte, el fisioterapeuta cumple un papel importante en la alteración del esquema corporal, definido como la etiqueta de las respuestas perceptivas y localizadoras referidas al propio cuerpo. Una alteración en la propia percepción del esquema corporal podría dar lugar a una experiencia de miembro fantasma después de sufrir una amputación de una de las extremidades inferiores. Esta experiencia constituye un claro argumento a favor de la existencia de un esquema corporal mental que subyace y modifica la experiencia del propio cuerpo ${ }^{26)}$. Sin embargo, muchas veces las alteraciones del esquema corporal no están asociadas con una amputación de la extremidad, sino con las alteraciones biodinámicas del pie, causada por el pie diabético. Alguna de estas alteraciones son las modificaciones del apoyo del pie, por la evolución del ser humano, y la utilización del calzado. En los diabéticos, la prevalencia de modificaciones del apoyo se observa en el $80 \%$ de los pacientes, que se ocasionan por la neuropatía motora, la osteoartropatía, la neuropatía sensitiva y la iatrogenia. Las 
alteraciones del apoyo, que se interrelacionan con la vasculopatía y la neuropatía, favorecen el desencadenamiento de una úlcera de pie, lo que finalmente puede llevar a una amputación de la extremidad. Por esta razón se ha considerado al pie diabético como una enfermedad biodinámica por el aumento de las presiones plantares, por las deformaciones, la limitación de la movilidad articular, las anormalidades de la marcha y el exceso de peso corporal ${ }^{(27)}$.

En este punto el fisioterapeuta tiene la responsabilidad de disminuir estos riesgos o de mejorar las limitaciones, alteraciones o deformidades del paciente; por ejemplo, al mejorar los arcos de movilidad articular, plantillas que repartan las cargas sobre la planta del pie, reeducación de la marcha y ejercicio aeróbico, entre otros.

\section{Fisioterapia en diabetes y pie diabético}

La fisioterapia se encarga del movimiento corporal humano como un elemento esencial de la salud y del bienestar del hombre, orientando sus acciones al mantenimiento, optimización o potencialización del movimiento, así como a la prevención y recuperación de sus alteraciones y a la habilitación y rehabilitación de las personas; para ello se apoya en conocimientos de ciencias biológicas, sociales y humanísticas que le dan sustento a su ejercicio profesional ${ }^{28)}$.

Es así como el modelo de atención fisioterapéutica propuesto por la American Physical Therapy Association (APTA) plantea que para el manejo del usuario/cliente es necesario realizar una serie de pasos interdependientes que conducen a una mirada integral del mismo y serán tenidos en cuentas a la hora de desarrollar esta propuesta.

El primero de ellos es la examinación que parte de la elaboración de la historia de salud e involucra la revisión de los sistemas relacionados con la generación del movimiento, principalmente cardiovascular y pulmonar, neuromuscular, integumentario y musculoesquelético, y finaliza con una toma de medidas y pruebas a fin de recoger toda la información relacionada con el paciente que, para el caso de la persona con diabetes y pie diabético, está comprometido, ya que esta enfermedad a largo plazo genera complicaciones como la neuropatía, las microangiopatías y las macroangiopatías responsables del pie diabético y la aparición de la enfermedad cardiovascular frecuente en este grupo poblacional.

Existen numerosas pruebas que nos indican el estado y funcionamiento de los nervios y los vasos sanguíneos en la persona con pie diabético, como son la valoración del aspecto, la temperatura, la coloración, la presencia de callos, deformidades articulares y secuelas de úlceras o cirugías previas del pie; palpación de los pulsos distales, pedios y tibiales posteriores y la exploración de la sensibilidad térmica, algesia y vibratoria, junto a la obtención del reflejo aquiliano, evaluación y caracterización del riesgo de ulceración, entre otros, en los cuales los fisioterapeutas nos apoyamos para el proceso de examinación ${ }^{(29)}$.

Después de recoger la información viene el proceso de evaluación, entendido como la organización y análisis de la información, teniendo en cuenta factores como el nivel de deficiencia, limitación y restricción, el estado general de salud, la disponibilidad de soporte social, el entorno vital, la cronicidad del problema, entre otros, todos importantes para definir los problemas del paciente que, en el caso del paciente diabético, la identificación del pie de riesgo es el elemento central de cualquier plan preventivo.

Es importante tener en cuenta las alteraciones biomecánicas ocasionadas por la neuropatía, lo que afecta la función motora, el sistema autónomo y la sensibilidad propioceptiva. Como consecuencia, la estática del pie se compromete (con distribución anormal de las presiones plantares) y de la dinámica (alteraciones en la marcha y en los puntos de apoyo), siendo necesario pensar en la evaluación y ulterior tratamiento del equilibrio en las personas diabéticas. Silva y colaboradores refuerzan el papel del fisioterapeuta desde la prescripción de ejercicios de fortalecimiento, flexibilidad, entrenamiento de marcha y equilibrio, además de la adaptación de prótesis y ortesis con el fin de prevenir la aparición de úlceras en los pies y reducir las secuelas originadas por el pie diabético ${ }^{(30)}$.

Una vez culminado este proceso se dará un diagnóstico fisioterapéutico, el cual organiza la información en términos de deficiencias que presenta el paciente, limitaciones de la actividad y restricciones en la participación en las cuales puede llegar a incurrir. Esto le permitirá al fisioterapeuta tener un punto de partida para la elaboración del pronóstico que busca obtener mejorías a corto plazo esperadas en distintos intervalos durante el curso de la intervención o la mejoría máxima que tal vez se logre y el tiempo requerido para alcanzar cada nivel, teniendo en cuenta factores como la seguridad, necesidades y objetivos del paciente, la anamnesis y el curso clínico esperado de la patología, deterioro o diagnóstico, los resultados de la exploración, evaluación y procesos diagnósticos ${ }^{(31)}$. Por último, se encuentra la realización del plan de tratamiento del paciente, donde el profesional de fisioterapia interactúa con diferentes métodos, técnicas y procedimientos a fin de lograr los objetivos planteados en el pronóstico. Para ello se puede recurrir al ejercicio físico/terapéutico y a la educación del paciente de manera preventiva, centrándose en el problema terapéutico.

Siendo la diabetes una enfermedad tanto para los médicos generales como para los especialistas en diversas ramas médicas que pasa, hasta cierto punto, inadvertida, tendemos a tolerar demasiado con ella y sus complicaciones debido a lo oculto en su curso. Sin embargo, a los fisioterapeutas nos llegan los pacientes diabéticos con complicaciones alarmantes, como lo es una amputación de una de sus extremidades infe- 
riores, causada por el deterioro de un pie diabético, por tanto, es importante conocer el manejo que se puede dar desde el servicio de fisioterapia, abarcando los tres niveles de atención:

\section{Prevención primaria}

En la prevención primaria durante el período preclínico, la educación terapéutica sobre cuidados del pie puede formar parte de un programa individual, como parte integral de la relación entre el paciente y el equipo integral de salud que trabaja con el paciente, de un programa de grupo o a través de los medios de comunicación, con el propósito de entender la importancia de la prevención, realizar la adecuada higiene e hidratación del pie, conocer la forma correcta e incorrecta de cortar las uñas, usar prendas y calzado adecuado, conocer los factores desencadenantes de lesiones (traumatismo mecánico, térmico, químico, deformidades) y actuar de forma adecuada para evitar la exposición, saber actuar ante la presencia de una herida y conocer cuándo deben asistir a consulta, saber la importancia del podólogo para el control de hiperqueratosis, deformidades y afección ungueal ${ }^{(32)}$.

La fisioterapia también tiene su importancia en este nivel de prevención al dirigir una actividad física planificada, previa a una evaluación realizada correspondiente del estado físico del paciente, a través de un programa de gimnasia específico donde no solo mejore la condición cardiopulmonar, sino que se enfatice en el aporte sanguíneo adecuado de miembros inferiores, mejorar o aumentar la fuerza muscular, movilidad articular, elasticidad, resistencia y, por ende, a un control metabólico de la enfermedad.

\section{Prevención secundaria}

Prevención secundaria durante el período clínico, en el cual se da un diagnóstico temprano y tratamiento inmediato. Tratamiento precoz de las infecciones, plantillas y órtesis y cirugía profiláctica del pie. El aporte de la fisioterapia en esta etapa tiene como objetivo mejorar la circulación con el objeto de aumentar la irrigación sanguínea en los miembros afectados, lo que retrasa el comienzo de la gangrena. Es conveniente también combatir el dolor. Puede mejorarse la circulación colateral y posiblemente reducirse el espasmo arterial. Como base del tratamiento y de acuerdo con las necesidades del paciente se realiza una evaluación fisioterapéutica antes de comenzarlo a ejecutar.

El tratamiento se basa en:

- El masaje para eliminar los productos de desecho de los tejidos y aliviar el espasmo. El masaje deberá ser sutil, con frotes suaves de deslizamiento, debe evitarse toda zona de dolor; se ha usado con éxito el masaje de tejido conjuntivo en algunos casos.

- Ejercicios correspondientes, especialmente en casos de claudicación intermitente sin gangrena los ejercicios de Buerguer. El paciente se encuentra en decúbito supino so- bre la mesa de tratamiento y realiza ejercicios de miembros inferiores, utilizando el efecto de la gravedad con intervalos de tiempo para provocar el estímulo de la circulación lenta y favorecer los cambios de presión en los vasos periféricos.

- Generalmente se aplica un tratamiento físico que produzca calor al abdomen, muslos o región lumbar. La sangre caliente de esta zona pasa al centro vasomotor, deprime su acción y produce relajación y dilatación de todos los vasos sanguíneos de las extremidades. El calor nunca debe aplicarse a los pies o a las piernas, ya que solo produce una vasodilatación local, la cual, sin un aporte sanguíneo, produce lesiones tisulares, tal es el caso de quemaduras por sensibilidad alterada.

Para que se lleve a cabo el proceso de cicatrización de los tejidos intervienen una serie de factores, entre ellos, el óptimo aporte de oxígeno, nutrientes y factores de crecimiento proporcionados mediante el torrente sanguíneo. Existen estudios que hablan del uso del láser de baja potencia y la corriente galvánica en pacientes con pie diabético, ya que estas modalidades terapéuticas ayudan a acelerar el proceso de cicatrización del tejido al colocar la corriente galvánica con el electrodo negativo cerca del área ulcerosa y el positivo a $25 \mathrm{~cm}$ distales de esta, lo que genera un incremento de la excitabilidad neuromuscular y vasodilatación activa, con ello se produce un aumento en el aporte de oxígeno y nutrientes y se origina una acción antiinflamatoria, antiedematosa y trófica de los tejidos; de igual forma, el uso de láser de baja potencia favorece y acelera la cicatrización de heridas. El mecanismo por el cual ocurre es debido a un aumento de fibroblastos, un incremento en la síntesis de procolágeno, mayor epitelización y menor tejido exudativo, así como mayor neoformación de vasos sanguíneos en el centro de la herida.

De la misma manera, se trabajarán ejercicios circulatorios de Buerguer Allen 2 veces al día con series en 3 fases (elevación, descenso y reposo) previa instrucción de paciente y acompañamiento de familiar. Vendaje compresivo de la extremidad afectada con el pie en dorsiflexión neutra o ligera dorsiflexión y eversión, comenzar a colocar la venda elástica distalmente y progresar hacia proximal para drenar el edema, vigilando signos de alteración circulatoria y masaje de derivación circulatoria (periherida). Se activan los grupos ganglionares más importantes de los miembros inferiores (inguinal, poplíteo y pedio) por medio de las maniobras de bombeo. Se realiza el masaje desde los artejos hasta la articulación coxofemoral, siempre de distal a proximal, concomitantes con otros tratamientos médicos y multidisciplinar (terapia de ozono y tratamiento farmacológico), por lo que se obtienen resultados favorables en la sintomatología y la pronta recuperación del miembro, esto mejora considerablemente la calidad de vida del paciente ${ }^{(33)}$. 


\section{Prevención terciaria}

Finalmente, en la prevención terciaria se inicia un proceso de rehabilitación adecuada de los pacientes que sufrieron amputaciones, para evitar deformaciones ulteriores y permitirle al paciente llegar a tener una máxima eficiencia con el mínimo de deficiencia al explotar sus capacidades dentro de sus limitaciones para llevarlo a una vida independiente, logrando su reincorporación a la sociedad gracias al trabajo de un equipo multidisciplinario integrado por diferentes profesionales de la salud, como médicos, enfermería, trabajo social, fisioterapia, terapia ocupacional, técnicos protesistas y psicología, esto mantiene una abierta comunicación y permite una exitosa rehabilitación del paciente ${ }^{(34)}$.

Desde la fisioterapia, como parte del equipo rehabilitador, el tratamiento de la persona amputada empieza por la evaluación de los antecedentes e historia quirúrgica del paciente amputado para conocer los niveles y tipos de amputación, seguido de una valoración física de las condiciones del muñón y de la funcionalidad del individuo. Se siguen los lineamientos de la APTA, la cual propone un plan de tratamiento con énfasis en la recuperación física usando principalmente el ejercicio y la educación. Se debe tener en cuenta el contexto donde se desenvuelve la persona y el apoyo familiar del mismo para que la integración al medio sea más fácil.

Asimismo, se puede apoyar en los diferentes protocolos y guías de manejo del paciente amputado que el equipo de trabajo considere. Existen propuestas que dividen el programa de rehabilitación en las siguientes fases:

- Fase prequirúrgica o preoperatoria

- Fase quirúrgica

- Tratamiento preprotésico

- Tratamiento protésico.

Además, la guía de práctica clínica para el diagnóstico y tratamiento preoperatorio, intraoperatorio y posoperatorio de la persona amputada, la prescripción de la prótesis y la rehabilitación integral también son de utilidad en la práctica diaria ${ }^{(35)}$. La guía para profesionales de la salud propone y da recomendaciones para las intervenciones en el paciente con diabetes, siendo de gran importancia la recomendación de realizar un programa de reacondicionamiento cardiovascular preoperatorio en pacientes mayores de 16 años con enfermedad vascular que estén en riesgo de amputación de miembros inferiores para mejorar la marcha y calidad de vida, en la cual el fisioterapeuta cuenta con los conocimientos y la habilidades para la prescripción del ejercicio físico. Igualmente, recomienda la implementación de un programa de rehabilitación física que incluya fuerza muscular, movilidad articular, equilibrio, marcha y reacondicionamiento físico en pacientes con amputación de miembro inferior, para mejorar la marcha, la movilidad y la adaptación protésica. También hace especial mención de la evaluación ocupacional y las adaptaciones ergonómicas en pacientes mayores de 16 años a quienes le fue amputado un miembro inferior por causas traumáticas, vasculares o por diabetes, para mejorar el funcionamiento y facilitar el retorno al trabajo o a una ocupación ${ }^{(36)}$.

De acuerdo con la valoración y condición del paciente se realizan las siguientes actividades: aprendizaje de la realización del vendaje del miembro residual para conseguir y dar la forma deseada al muñón; ejercicios de fortalecimiento para la extremidad afectadas; ejercicios para ampliar y conservar el arco articular y de sensibilización del muñón; ejercicios de corrección postural frente al espejo; entrenamiento de marcha entre paralelas; entrenamiento de la marcha con banquillos, muletas y otros aditamentos utilizados para la marcha dentro y fuera de paralelas; cuclillas con el miembro indemne y entrenamiento del equilibrio ${ }^{(35)}$.

También es importante inculcar la implementación de la actividad física en los individuos sanos y con DM y PD, pues no solo se ejercita el cuerpo, incluso se obtienen grandes beneficios para la salud y ayuda a prevenir las enfermedades no transmisibles (ENT). Según cifras de la OMS, la inactividad física es uno de los principales factores de riesgo de padecer ENT, como las enfermedades cardiovasculares, el cáncer y la diabetes, ocupando el cuarto lugar entre los principales factores de riesgo de mortalidad a nivel mundial. Por esta razón, el paciente con diabetes al realizar ejercicio mejora el metabolismo de la glucosa a varios niveles: páncreas, músculo y tejido graso; ayuda también a controlar el peso, los niveles de glucosa, presión arterial, colesterol y otras alteraciones asociadas con la diabetes, por tanto, en las personas que tienen diabetes o riesgo de padecerla hacer ejercicio es aún más importante.

Múltiples estudios que demuestran el beneficio del ejercicio regular en el control glucémico a largo plazo han utilizado la actividad física (AF) realizada por 30 a 60 minutos, al $50 \%$ a $80 \%$ del consumo de oxígeno máximo $\left(\mathrm{VO}_{2}\right)$ de 3 a 4 veces por semana. Con este tipo de programas se logran reducciones de $10 \%$ a $20 \%$ en la hemoglobina glucosilada ${ }^{(24)}$. También es importante resaltar que el ejercicio solo sin restricción calórica y sin modificación de la conducta tiende a alcanzar solo modestas pérdidas de peso de aproximadamente $2 \mathrm{~kg}$, logrando obtener un control de peso a largo plazo con ayuda de una correcta dieta.

No solo es importante conocer los tres niveles de atención y los beneficios del ejercicio, sino también saber que la educación en un paciente diabético es obtener y mantener conductas que lleven a un óptimo manejo de la vida con la enfermedad. Las personas con diabetes deben adquirir conocimientos y desarrollar destrezas, además tomar diariamente decisiones relativas a modificar su estilo de vida. Los servicios de salud públicos y privados deben entregar programas de educación en diabetes desde que el paciente se ha diagnosticado con esta patología hacia adelante; además, hay que considerar que 
todos los escenarios pueden ser una buena instancia para la educación en diabetes: una hospitalización, la consulta con el podólogo, visita al dentista, entre otras.

La American Association of Diabetes Educators (AADE) propone 7 conductas de autocuidado para las personas con diabetes ${ }^{(37)}$ :

- Comer sano

- Ser físicamente activo

- Monitorización

- Toma de medicamentos

- Resolución de problemas

- Reducción de riesgos

- Afrontamiento saludable.

\section{Conclusiones}

La diabetes y el pie diabético continúan siendo un problema de salud pública a pesar de los avances en el conocimiento del mismo y de las campañas de prevención tendientes a reducir los factores de riesgo que lo ocasionan. Esta situación preocupa a los profesionales de la salud, en quienes está la responsabilidad de mejorar la calidad de vida de las personas y de la comunidad. Aunque la ciencia ha avanzado en el manejo de la diabetes, especialmente desde el área farmacológica y desarrollo tecnológico, continúa presentando debilidad frente a una estrategia sencilla y costo efectiva, como lo es la educación de la persona diabética y su familiar en cuanto a los cuidados sobre diabetes y prevención del pie diabético.

La fisioterapia, siendo una profesión que aborda al individuo, la familia y la comunidad, debe ampliar sus procesos de intervención a la educación de los mismos con el ánimo de contribuir a la reducción de esta problemática.

\section{Agradecimientos}

Ft. Jhovanna Olmos B. - Mg. SO. Docente Programa de Fisioterapia- USC.

Flgo. Carol Vallecilla - Coordinadora Celoa USC, seccional Palmira.

Ft. Luz Angela Reyes L.- Mg Educación Desarrollo Humano, Docente Programa de Fisioterapia, USC Palmira.

Charfuelan PA; Díaz C; Muñoz DC; Paredes JD; Daza LJ; Quiceno D; Monsalve SG.; De Castro N; Cardozo DK; Valencia MF; Vásquez MA; Caicedo JA; Ortiz JA; Saavedra C; Plaza ED; Palacio LL; Echeverry E; Zapata LM; Melo MA; estudiantes integrantes del semillero de investigación período 2016B.

\section{Referencias}

1. Paiva O, Rojas N. Pie diabético: ¿podemos prevenirlo? Rev Med Clin Condes. 2016;27(2):227-34.

2. Organización Mundial de la Salud. Informe Mundial sobre la Diabetes. Resumen de Orientación. Suiza. 2016.

3. Proenza L, Figueredo R, Fernández S, Baldor Y. Factores epidemiológicos asociados al pie diabético en pacientes atendidos en el Hospital Celia Sánchez Manduley. Rev Multimed. 2016:543-56.

4. Alonso M, de Santiago A, Moreno A, Carramiñana FC, López F, Miravet S, et al. Guías clínicas diabetes mellitus. Barcelona: euromedice. 2016.

5. Silva P, Rezende M, Ferreira L, Dias F, Rodrigues H, Silveira C. Cuidados de los pies: el conocimiento de las personas con diabetes mellitus inscritos en el programa de salud familiar. Enferm Global. 2015:30-50.

6. Organización Mundial de la Salud. 1 de cada 11 personas en el mundo ya tiene diabetes. BBC Mundo. 2016. Disponible en: https://www.bbc.com/ mundo/noticias/2016/04/160406_salud_diabetes_oms_lb.

7. Observatorio Nacional de Salud. Carga de enfermedad por enfermedades crónicas no transmisibles y discapacidad en Colombia. Bogotá. 2015.

8. Rosales M, Bonilla J, Gómez A, Gómez C, Pardo J, Villanueva L. Factores asociados al pie diabético en pacientes ambulatorios. Centro de Diabetes Cardiovascular del Caribe. Barranquilla (Colombia). Rev Cient Salud Uninorte. 2012;28(1).

9. Tamayo D. Diabetes en Colombia: costos asociados con su cuidado. Observatorio Diabetes Colom. p. 4-8.

10. Análisis De Situación De Salud (ASIS) Colombia, 2017; Dirección de Epidemiología y Demografía Bogotá; disponible en: https://www.minsalud. gov.co/sites/rid/Lists/BibliotecaDigital/RIDE/VS/ED/PSP/asis-nacional-2017.pdf.

11. Romero Prada ME, Pajaro A, Alfonso Quiaones PA. Impacto económico de la hipoglucemia en Colombia [internet]. Value in Health; Elsevier; 2017. Disponible en: https://www.valueinhealthjournal.com/article/S10983015(17)33201-1/pdf.
12. Asteazaran S, Elgart J, Gagliardino J. Impacto de la diabetes sobre la productividad en Argentina. Value Health. 2003;16:A665-728.

13. Briones E. Ejercicios físicos en la prevención y control de la diabetes mellitus. Dom Cien. 2016;2:47-57.

14. Bakker K, Riley P. El año del pie diabético. Diabetes Voice. 2005;50(1):11-4.

15. Del Castillo R, Fernández J, Del Castillo F. Guía de práctica clínica en el pie diabético. Arch Med. 2014;10(2).

16. Pinilla A, Barrera M, Sánchez A, Mejía A. Factores de riesgo en diabetes mellitus y pie diabético: un enfoque hacia la prevención primaria. Rev Colomb Cardiol. 2013;20(4):213-22.

17. Hernández J, Arnold Y, Mendoza J. Efectos benéficos del ejercicio físico en las personas con diabetes mellitus tipo 2. Rev Cub Med Fis Rehab. 2018;29(2).

18. Ministerio de Salud. Guía de atención de la diabetes tipo II. Ministerio de Salud - Dirección General de Promoción y Prevención. 2014. p. 7-12.

19. Pinilla A, Barrera M, Sánchez A, Mejía A. Factores de riesgo en diabetes mellitus y pie diabético: un enfoque hacia la prevención primaria. Rev Colomb Cardiol. 2013;20(4):213-22.

20. Hernández J, Lozano G, Murillo V. Experiencias de educación para la salud en fisioterapia. Rev Univ Salud. 2016;18(3).

21. Ibáñez V, Martín JL, Vázquez P. Estrategias de prevención en atención primaria y hospitalaria [internet]. Disponible en: http://www.sld.cu/galerias/pdf/sitios/rehabilitacion/capitulo_13.pdf.

22. Nicolás P, Hyunsuk P, Hong J. Úlceras del pie diabético: importancia del manejo multidisciplinario y salvataje microquirúrgico de la extremidad. Rev Chil Cir. 2018;70(6):535-43.

23. Cisneros N, Ascencio I, Vita N, Rodríguez H, Campos A, Dávila J, et al. Índice de amputaciones de extremidades inferiores en pacientes con diabetes. Med Inst Mex Seguro Soc. 2016:477-8. 
24. Márquez JJ, Suárez R, Márquez J. El ejercicio en el tratamiento de la diabetes mellitus tipo 2. Rev Argent Endocrinol Metab. 2012;49:203-12.

25. Quilez P, García M. Control glucémico a través del ejercicio físico en pacientes con diabetes mellitus tipo 2; revisión sistemática. Nutr Hosp. 2015;31(4):1465-72.

26. Huertas R. Neuroplasticidad asociada a miembro fantasma. Rev Soc Esp Dolor. 2014; 21(6):345-50.

27. Fundación Escuela para la formación y actualización en Diabetes y Nutrición. Alteraciones biodinámicas del pie. 2011. Disponible en: http://www. fuedin.org/articulos_fuedin/detalle.php?articulo=161

28. Congreso de Colombia. Ley 528 de 1999. Diario Oficial.

29. Gómez E, Levy E, Díaz A, Cuesta M, Montañez C, Calle A. Pie Diabético. Semin Fund Esp Reumatol. 2012;119-29.

30. Silva VC, Dantas CD, Moreira RE, Maia RH. Fisioterapia e neuropatias periféricas em portadores de diabete melito II: produção bibliográfica entre 1966 e 2011. Rev Fisioter S Fun Fortaleza. 2012;1(1):47-51

31. Hall CM, Brody LT. Ejercicio terapéutico: recuperación funcional. Barcelona: Paidotribo. 2006.
32. Pereira O, Palay M, Ríos V, Neyra R. Efectividad de un programa educativo en pacientes con pie diabético de riesgo. MEDISAN. 2015;19(1)

33. Rivera A, Llaguno A, Chuliá N. Pie diabético. Tratamiento médico multidisciplinario y Ozonoterapia. Rev Esp Ozonoterapia. 2018;8(1).

34. Díaz M. Pie diabético: un enfoque desde la fisioterapia. Disponible en: https://grupoangeles.org/espanol/pie-diabetico-un-enfoque-desde-lafisioterapia-licda-en-rehabilitacion-monica-diaz

35. Govantes $\mathrm{Y}$, Alba $\mathrm{C}$, Arias A. Protocolo de actuación en la rehabilitación de pacientes amputados de miembro inferior. Rev Cub Med Fis Rehab. 2016;8(1):33-43.

36. Ministerio de Salud y Protección Social. Guía de práctica clínica para e diagnóstico y tratamiento preoperatorio, intraoperatorio y postoperatorio de la persona amputada, la prescripción de la prótesis y la rehabilitación integral. Ministerio de Salud y Protección Social. 2015.

37. Hevia V. Educación en diabetes. Rev Med Clin Condes. 2016;27(2):271-6. 